\title{
Pathologische Frakturen bei Osteomyelitis/Osteitis
}

\author{
- Thomas von Stein, Volker Bühren
}

\section{Zusammenfassung}

Pathologische Frakturen im Rahmen einer Osteomyelitis/Osteitis sind in der Regel Folge- oder Refrakturen. Die heutzutage selten gewordene, vorwiegend juvenile Osteomyelitis führt nur noch selten zu Spontanfrakturen, beim älteren Erwachsenen sind nahezu ausschließlich die Wirbelkörper betroffen. Grundvoraussetzung einer erfolgreichen Therapie der Osteomyelitis ist die Sanierung des Knocheninfektes. Im Rahmen der Rekonstruktion und erneuten Stabilisierung ist unbedingt darauf $\mathrm{zu}$ achten, dass vitaler Knochen mittels möglichst stabiler Osteosynthese verbunden oder rekonstruiert wird. In der Nachbehandlung sollten dystrophiebegünstigende Faktoren wie z. B. eine lange Immobilisierung und Entlastung vermieden werden. Die Knochenbruchheilung nach infektbedingter pathologischer Fraktur verläuft individuell höchst unterschiedlich. Ein Regelzeitintervall zur ggf. notwendigen Metallentfernung kann daher nicht angegeben werden und ist stets individuell radiologisch zu prüfen.

\section{Pathological Fractures and Osteomyelitis/Osteitis}

Pathological fractures complicating acute or chronic osteomyelitis are usually refractures. Currently, the typical juvenile osteomyelitis causes spontaneous fractures only rarely whereas in elder adults osteomyelitis affects the vertebral bodies predominently. As a basic principle, the first step of the therapy must be decontamination of the bone infection. Within the limits of reconstruction and stabilisation, it is necessary to connect vital bone. During rehabilitation process long periods of immobilisation and absence of load-bearing should be avoided. The healing of the bone after pathological fractures caused by infection proceeds very individually. Therefore, the date for removal of the osteosynthesis, if necessary, depends mainly on the results of X-ray check-ups.

\section{Einleitung}

Definitionsgemäß handelt es sich bei pathologischen Frakturen infolge Osteomyelitis/Osteitis um Frakturen, die ihre Ursache in einer krankhaften Veränderung der Knochenstruktur haben und den Knochen meist schon durch sog. inadäquate Unfallmechanismen, also bei geringer Krafteinwirkung, brechen lassen.

OP-JOURNAL 2006; 22: 10-15

(c) Georg Thieme Verlag KG Stuttgart · New York
Neben der direkten Zerstörung des Knochens kann der Schädigungsmechanismus auch in einer Rarifizierung (Verarmung) der knöchernen Struktur ähnlich der Osteoporose, der Dystrophie durch Immobilisierung oder der chronischen Osteitis/Osteomyelitis bestehen. Dadurch wird der Knochen zwar härter, er sklerosiert, büßt aber dafür erheblich an Elastizität ein, das heißt er wird spröde, wodurch er wiederum deutlich leichter frakturiert $[7,10]$.

Zugrunde liegt in aller Regel ein Ungleichgewicht im Knochenstoffwechsel (Knochenabbau, -aufbau, - umbau).
Ziel der jeweiligen Therapie, ob konservativ oder operativ, muss es sein, die Reparaturmechanismen derart günstig $\mathrm{zu}$ beeinflussen, dass letztlich eine knöcherne Heilung ad integrum, also mit einem normalen Knochenbau und damit Stabilisierung eintreten kann.

\section{Entstehung}

Im deutschsprachigen Raum wird im Gegensatz zum angelsächsischen Sprachraum zwischen Osteomyelitis und Osteitis unterschieden. Von beiden Erkrankungen existiert jeweils eine akute und eine chronische Form. Eine Knochenentzündung gilt als chronisch, wenn sie länger als 6 Wochen besteht.

Klinisch sinnvoll ist hierbei die Unterscheidung zwischen unmittelbar infektbedingten Zerstörungen des Knochens, die zu Frakturen führen und andererseits den strukturellen Veränderungen des Knochens, die auch nach sanierter Entzündung des Knochens verbleiben.

\section{Knochendestruktion}

Sowohl innere, endogene als auch äußere, exogene Ursachen können zu einer zerstörenden Entzündung des Knochens und nachfolgender Fraktur führen.

\section{Endogene Ursachen}

Die typische Ursache einer endogenen, infektbedingten Zerstörung des Knochens ist die Osteomyelitis.

Die Osteo-myelitis ist eine bakterielle Infektion des Knochens, die endogen, also hämatogen oder lymphogen, ohne äuBere Eintrittspforte entsteht.

Die Osteomyelitis beginnt von innen und breitet sich nach außen fort. In der Regel liegt ein bakterieller Herd, wie z. B. eine Sinusitis oder Tonsillitis, zugrunde. Diese Erkrankung betrifft vorwiegend Kinder und Jugendliche, wobei die vorwiegend 

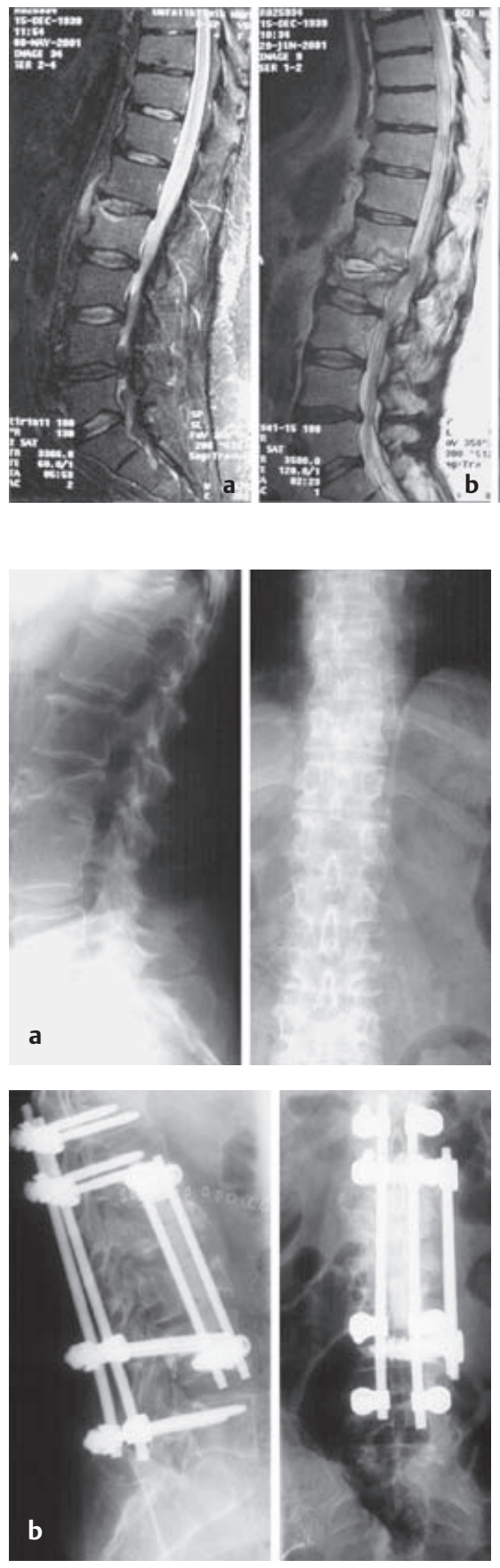

Abb. 2 (a) 78-jährige Frau, pathologische Fraktur L3 bei tuberkulöser Spondylitis L2/3. (b) nach Teilkorporektomie dorsoventrale Stabilisierung mit winkelstabilem Stabsystem und Knochenzement-Platzhalter.

betroffenen Skelettanteile das Femur und der Unterkiefer sind. Im älteren Erwachsenenalter, häufig bei begleitendem Diabetes mellitus, ist vorwiegend die Wirbelsäule betroffen (Abb.1).

Die jugendlichen Osteomyelitiden sind in den westlichen Ländern stark zurückgegangen und verursachen daher eher selten pathologische Frakturen. In den

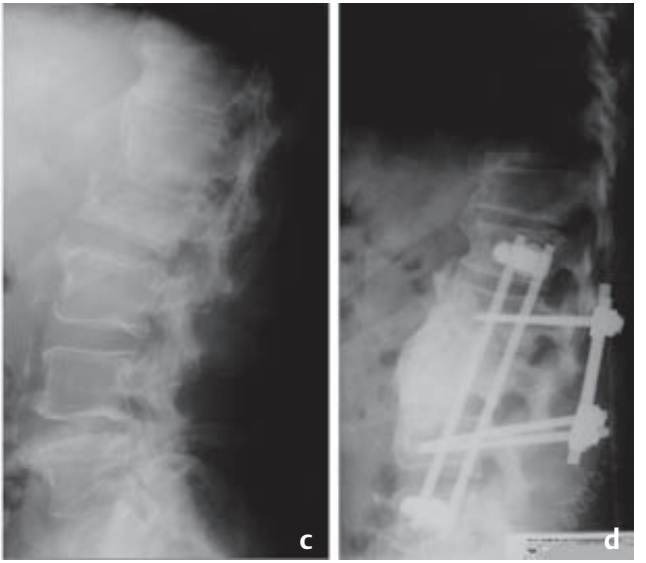

Abb. 1 (a) MRT, 62-jähriger Mann mit starken Schmerzen im thorakolumbalen Bereich, beginnende Spondylitis L1 und V.a. Abszess prävertebral. (b, c) MRT, Röntgen, trotz antibiotischer Therapie fortschreitende Destruktion und pathologische Kompressionsfraktur L1, mitbetroffen bereits Bandscheibe und Th12. (d) nach Teilkorporektomie dorsoventrale Stabilisierung mit winkelstabilem Stabsystem und Knochenzementplombe.
Ländern der Dritten Welt spielt diese Erkrankung aber noch eine erhebliche Rolle [11]. Der mit Abstand häufigste Erreger dieser Erkrankung ist der Staphylococcus aureus. Andere Erreger spielen eine eher untergeordnete Rolle. Durch die europäische Öffnung nach Osten muss jedoch zunehmend wieder an die Tuberkulose als mögliche Ursache gedacht werden (Abb.2) [12].

\section{Exogene Ursachen}

Entsteht durch äußere Einwirkung eine Infektion am Knochen, so wird diese als Osteitis bezeichnet.

Häufigste Ursache ist die offene Fraktur, gefolgt von operativen Eingriffen am Knochen. Weitere Ursachen können unmittelbare oder mittelbare Schädigungen der Weichteile sein, wie z.B. ein Quetschtrauma, eine diabetische bzw. durch eine periphere arterielle Verschlusskrankheit bedingte Gangrän oder ein chronisches Druckulkus. Bei der Osteitis spielt der Weichteilmantel eine wesentliche Rolle, was sich unter anderem in der Therapie niederschlägt. Je besser die muskuläre Deckung und je geringer die Deperiostierung des Knochens ist, um so besser ist die Durchblutung, und das Infektionsrisiko sinkt. Am häufigsten finden sich posttraumatische und postoperative Osteitiden im Bereich des distalen Unterschenkels, da hier die Durchblutung, bedingt auch durch die geringe Weichteildeckung, am ungünstigsten ist.

Eine exogen verursachte infektiöse Destruktion des Knochens kann z.B. im Rahmen einer Versorgung mittels Fixateur externe entstehen. Über die Pins gelangen die Erreger an den Knochen und verursachen hier eine entsprechende De- struktion, die im ungünstigen Fall zu einer Fraktur führt (Abb.3).

Auch bei der Osteitis ist der häufigste Erreger der Staphylococcus aureus. Das Keimspektrum ist jedoch deutlich breiter als bei der Osteomyelitis [12], weitere häufig zu findende Erreger sind Staphylococcus epidermidis, Enterokokken und Pseudomonaden.

\section{Folgezustände nach sanierter Knocheninfektion}

Der größte Teil infektbedingter pathologischer Frakturen sind Folge- oder Refrakturen, die in der Regel nach sanierter Osteitis/Osteomyelitis auftreten.

Als Folgezustand der infektbedingten Vorschädigung verbleiben chronische Veränderungen des Knochens, die nur sehr langsam oder gar nicht ausgeglichen werden können. Zum einen können Knochendefekte verbleiben, die zu einer mechanischen Schwächung der Stabilität führen, zum anderen resultieren strukturelle Veränderungen der Knochenqualität, die über Veränderungen der Elastizitätseigenschaft ebenfalls zur verminderten Frakturresistenz führen.

\section{Infektbedingte Störungen der Frakturheilung}

Bei der Osteitis nach offener Fraktur oder Eingriffen am Knochen kommt es durch Infektion, Weichteilschaden und Durchblutungsstörung zur Dystrophie des Knochens und zu einer gestörten Frakturheilung.

- Durch die Entzündung ist das Gleichgewicht zwischen Knochenabbau und Knochenaufbau gestört, es entstehen osteolytische Defekte. 

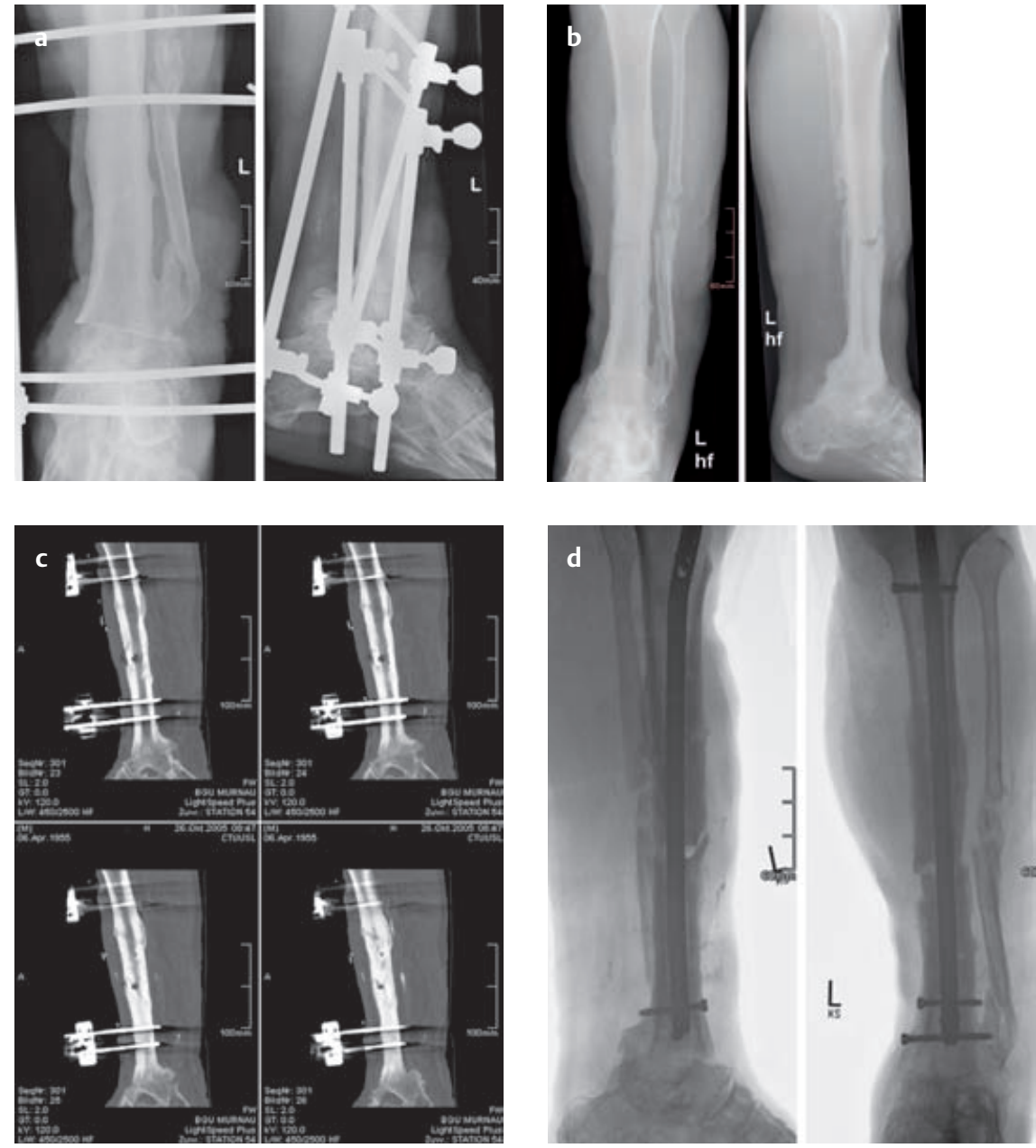

Abb.3 (a) 50-jähriger Mann, OSG-Arthrodese mit Fixateur externe bei posttraumatischer Arthrose, Tibia-Osteitis 5 Jahre nach offener US-Fraktur. (b) Fraktur durch persistierenden Pininfekt 2 Monate nach Fixateurentfernung, Defekt gut sichtbar. (c) Erstversorgung mit Fixateur externe in anatomischer Stellung und Infektsanierung. (d) 6 Monate später bei ausbleibender Frakturheilung Anfrischen der Frakturenden und Versorgung mit Kompressionsmarknagel.

- Sklerosierung und verminderte Elastizität des Knochens sind die Folge.

- Nekrotische oder von der Blutversorgung abgeschnittene Knochenareale werden nicht revitalisiert und bleiben als avitale Fragmente, also Sequester, liegen. Nekroseareale können sich vergrößern. Die Infektion wird unter anderem durch die nekrotischen Weichteil- und Knochenanteile aufrechterhalten und chronifiziert, was wiederum zur Störung der Frakturheilung führt. Verbliebene Implantate, an denen Bakterien besonders gut haften, sind häufig die Ursachen für das Persistieren eines Knocheninfektes [4].

\section{Mechanische Störfaktoren}

Im Rahmen der verzögerten Bruchheilung nach sanierter Osteitis spielen mechanische Ursachen eine wesentliche Rolle.

Pathophysiologisch werden verschiedene Bruchformen beschrieben.

\section{Kallusbruch}

Der Kallusbruch entsteht bei plötzlicher Überbelastung eines unter Kallusbildung heilenden oder geheilten Bruches. Er tritt meist in einer Phase der Knochenbruchheilung auf, in der eine volle Belastungsstabilität noch nicht gewährleistet ist und wird durch eine ungewollte oder unkontrollierte Belastung der Extremität ausgelöst.

\section{Ermüdungsbruch}

Der Ermüdungsbruch wird ursächlich durch eine andauernde Überbeanspruchung des Knochens oder des Metallimplantates oder beider Komponenten hervorgerufen. Unphysiologische Beanspruchung des neu gebildeten Knochengewebes über einen längeren Zeitraum führt zu einem pathologischen Umbau der Knochenstruktur. Der Knochen verliert in diesem Bereichen seine elastischen Eigenschaften und wird spröde. Vergleichbar hierzu sind Materialermüdungen und deren Brüche. Durch die Wechselbiegebelastung kommt es zur Zermürbung des Metallgefüges mit daraus resultierendem Implantatbruch. Demzufolge sind solche Implantatversagen meist Zeichen einer gestörten bzw. ausbleibenden Frakturheilung.

\section{Neutralisationsbruch}

Die funktionelle Anpassung des Knochens an das Osteosynthesematerial stellt die Ursache für den Neutralisationsbruch dar. Je mehr die auf den Knochen einwirkenden Kräfte durch das Implantat neutralisiert werden, desto stärker wird der Knochen entlastet und atrophiert. Es kommt zu einer Porosierung der Korrtikalis unter dem Metall. Nach der Implantatentfernung bricht der mechanisch geschwächte Knochen bereits bei geringer Belastung.

\section{Dystrophiebruch}

Ursache des Dystrophiebruches ist die lokale gestörte Durchblutung des Knochens. Infolge einer infektbedingten und einer additiv unfallbedingten oder operativen Schädigung kann es zu einer Minderversorgung des Knochens kommen, die die Konsolidierung des Bruches verändert. Dabei kann sowohl die endostale als auch die periostale Blutzufuhr gestört sein. Klinisch manifest wird die Mindervorsorgung, wenn beide Komponenten der Blutzufuhr betroffen sind. Aber auch eine zu lange Ruhigstellung und Entlastung sind aufgrund der konsekutiven Minderdurchblutung des Knochens dystrophiebegünstigend. Eine besondere Bedeutung kommt zusätzlich der Integrität der Weichteile und der Innervation zu. Läsionen in diesen Bereichen fördern auf neurovaskulärem Wege verzögerte Knochenheilung. Dystrophiefrakturen können auch nach geringen Traumen im gesamten geschädigten Gebiet auftreten und müssen nicht in räumlichen Bezug zur Erstfraktur stehen [4, 7] (Abb.4). 
Bei den septischen Folgefrakturen bestehen neben der Dystrophie (100\%) als wesentliche Veränderungen Spongiosierungen (80\%), Kortikaliskerben (55\%), Schraubenlöcher (25\%), Sequester (25\%) und Ermüdungen (10\%). Fast immer wirken mehrere dieser Faktoren bei den septischen Folgefrakturen zusammen [7].

\section{Klinik/Diagnostik}

Klinisch stehen Schmerz, Schwellung und Belastungsunfähigkeit im Vordergrund.

Zeichen einer ursächlichen Osteitis sind auffallende Rötung, chron. Hautveränderungen, Fisteln, putride Sekretion. Eine Erhöhung der Leukozyten ist unspezifisch, sie tritt auch bei der akuten traumatischen Fraktur auf. Deutliche CRP-Erhöhung und insbesondere ein erhöhtes PCT (Procalcitonin) deuten auf eine bakterielle Infektion hin. Radiologisch zeigen sich Osteolysen, perifokale Verknöcherungen (Involucrum), ungerichtetes Knochenwachstum und erhebliche Sklerosierung. Häufig finden sich Sequester, die im Zweifelsfall deutlich besser in der Computertomographie (CT) zu sehen sind.

Beweisend für die infektiöse Genese der Fraktur ist der Erregernachweis in der mikrobiologischen Untersuchung.

Zur Sicherung der Diagnose und zum Ausschluss z. B. maligner Ursachen sollte intraoperativ im Rahmen des Ersteingriffes stets eine Histologie abgenommen werden [9].

\section{Therapie}

\section{Akute Infektion}

Liegt die Ursache einer pathologischen Fraktur in einem akuten Infekt, sei es eine Osteomyelitis, eine akute Osteitis oder ein akutes Wiederaufflackern einer chronischen Osteitis, so steht zunächst die Sanierung des Infektes im Vordergrund.

Immer ist dies im Bereich der Extremitäten in erster Linie eine operative Therapie, die von einer Reihe adjuvanter Therapieformen begleitet wird.

Die Infektsanierung der akuten Osteitis erfordert zunächst das ausreichende Débridement des betroffenen Knochens und der umgebenden Weichteile und
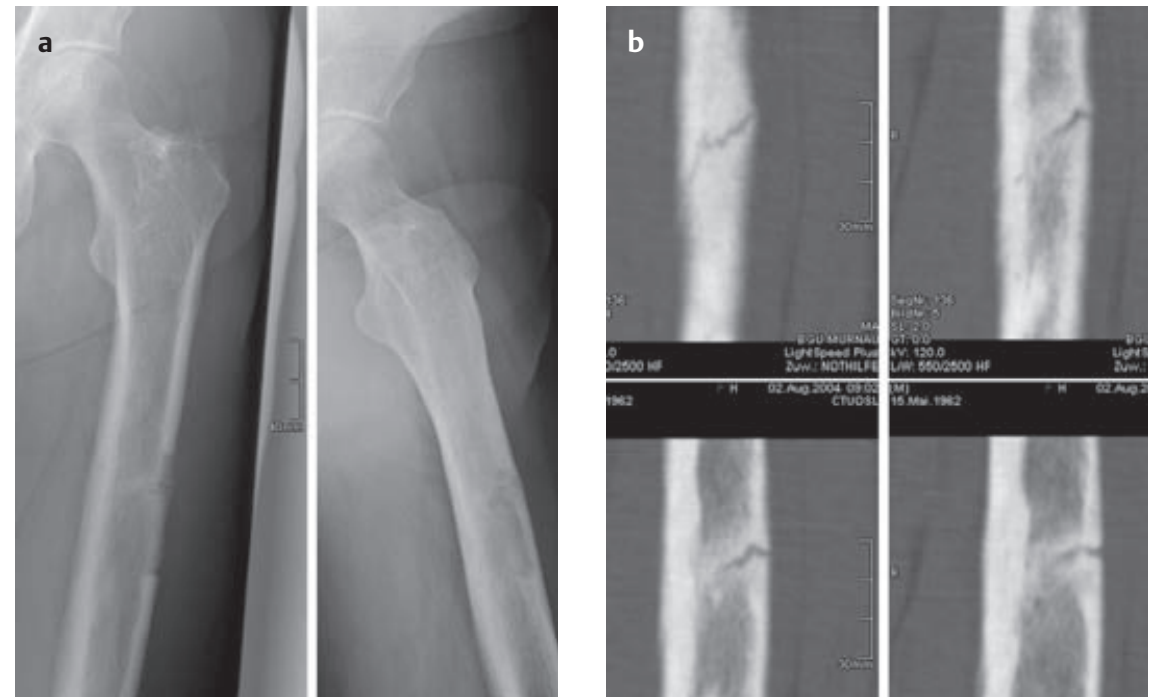

wird im Rahmen eines sog. programmierten Etappenlavageprogramms durchgeführt $[5,11]$. Dabei werden zunächst verbliebene Fremdkörper, wie PMMAKetten, Palacosreste, Implantate, abgebrochene Schrauben etc., entfernt. Mindestens zweimal wöchentlich werden infizierte und nekrotische Knochen- und Weichgewebsanteile debridiert und der Situs ausgiebig mit Ringer gespült. Die Anwendung von Jetlavagen (gepulste Hochdruckspülung) hat sich im Bereich knöcherner Strukturen bewährt, kann aber im Bereich der Weichteile durch ein iatrogenes Ödem kontraproduktiv sein. Zusätzliche antiseptische Spülungen (z.B. Octenisept, Lavasept) können durchgeführt werden, bergen aber die Gefahr einer systemischen Einschwemmung und daneben einer Unverträglichkeitsreaktion. Die Einlage von testgerechten lokalen Antibiotikaträgern, wie PMMAKetten, Gentamycin- oder VancomycinKollagen-Vliesen, führt vor Ort zu hohen Wirkspiegeln, ersetzt jedoch die obligate systemische testgerechte Antibiotikumtherapie nicht. Der vorübergehende Wundverschluss sollte über eine Vakuumversiegelung mittels Polyurethanschwamm erfolgen [1, 3]. Dieser führt zum einen das entstehende Wundsekret ab und bewirkt andererseits eine Stoffwechselverbesserung der gesamten Wunde mit all ihren Vorteilen, wie z.B. vermehrte Granulation, verbesserte Durchblutung, bessere Wirksamkeit der systemischen Antibiotikumtherapie. Gleichzeitig muss zur Schmerzausschaltung und zur Beruhigung des Infektgeschehens für eine ausreichende Stabilisierung der Extremität gesorgt werden. Am günstigsten ist hier die Lagerung in einem maßgefertigten Hartstoffverband.

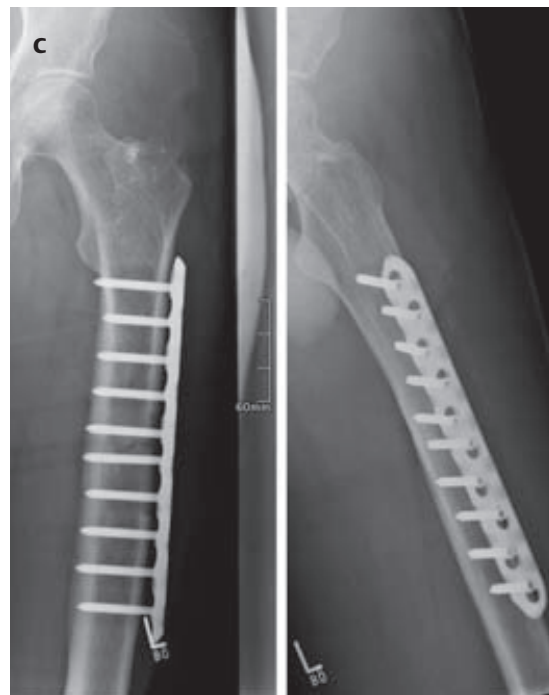

Abb.4 (a,b) 43-jähriger Mann, OS-Fraktur mit 10 Jahren, Infektverlauf über 5 Jahre, 20 Jahre beschwerdefrei, Sprödbruch 1 Monat nach PE einer chron. Osteitis bei Schmerzen und V. a. Osteitisrezidiv, bakteriologisch keimfrei, in der CT sind die Fraktur und die Sklerosierung deutlich erkennbar. (c) Versorgung mit winkelstabiler Titanplatte.

Ist dies nicht möglich, so wird eine äußere Stabilisierung mittels Fixateur externe oder eine innere Stabilisierung notwendig werden. Die Stabilisierung mittels Fixateur externe verhindert in den meisten Fällen ein ausreichendes Markraumdébridement, so dass ggf. auf eine innere Stabilisierung mittels Spacer (Platzhalter) oder jeweils zu wechselndem Marknagel (resterilisierbar) zurückgegriffen werden muss.

In schwerwiegenden Fällen müssen avitale Knochenanteile im Sinne einer Segmentresektion entfernt und nach erfolg- 

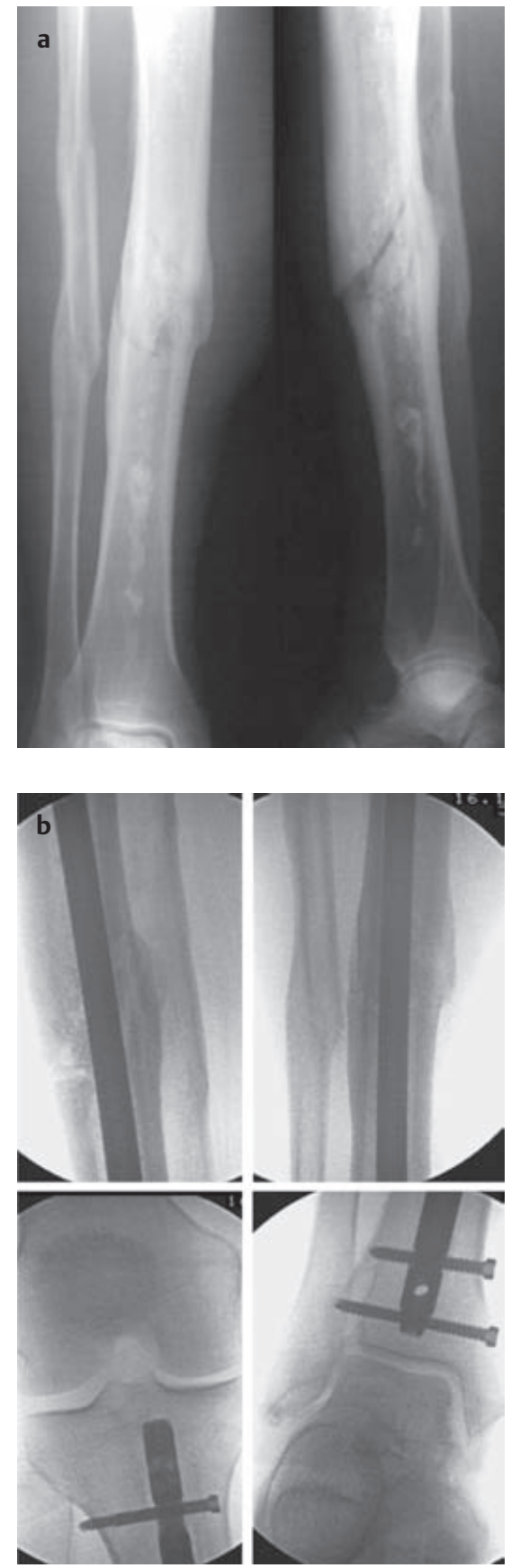

Abb.5 (a) 47-jähriger Mann, Sprödfraktur 12 Jahre nach infizierter US-Fraktur ohne Infektrezidiv, gut sichtbar die erhebliche Sklerosierung und Verplumpung. (b) Aufbohren und Kompressionsmarknagelung mit Titannagel. ter Sanierung wieder rekonstruiert werden. Gelegentlich ist infolge eines nicht therapierbaren chronifizierten Infektes auch eine Amputation notwendig.

Neben der adjuvanten antibiotischen Therapie kann, je nach Lokalisation, eine begleitende HBO-Therapie (hyperbare Sauerstofftherapie) zur Anwendung kommen [13].

\section{Therapie der Sprödfraktur}

Im Rahmen der Frakturversorgung muss dafür Sorge getragen werden, dass möglichst wieder heilfähiger und damit belastbarer Knochen entsteht.

Dies gelingt am ehesten, indem eine möglichst stabile, wenig durchblutungskompromittierende Osteosynthese zur Anwendung kommt. In wenigen Fällen, bei sicher vitalen Frakturenden, wird dies z. B. bereits durch das Aufbohren einer Schaftfraktur und eine nachfolgende Marknagelung gelingen [14] (Abb.3,5).

Die Durchblutungsverhältnisse sollten optimiert werden. Bisweilen sind zur entsprechenden Weichteildeckung lokale oder freie Lappenplastiken notwendig. Die Frakturenden, die in einem dystrophen Knochenbereich liegen, müssen angefrischt werden, so dass vitaler, durchbluteter Knochen in Kontakt kommt. Hier müssen unter Umständen Verkürzungen in Kauf genommen werden. Ist der Defekt zu ausgedehnt, kommen eine Spaninterposition, ein freier osteomyokutaner Transfer oder eine ausgedehnte Spongiosaplastik infrage [2]. Bei kortikalen Defekten über 3-4cm wird ein Segmenttransport zur Kallusdistraktion notwendig, alternativ kann ein gestielter Knochentransfer (z. B. Fibula) in Betracht gezogen werden. Zur ggf. notwendigen schnelleren Mobilisierung ist auch ein Knochenteilersatz durch sog. modulare Ersatzimplantate möglich, insbesondere an Humerus und Femur.

Wesentlich ist die stabile, durchblutungsschonende Osteosynthese, die vor allem die periostale Durchblutung respektieren soll.

Hier zeichnen sich neben der MarknagelOsteosynthese die winkelstabilen Implantate aus, da diese nicht unmittelbar an den Knochen gezogen werden müssen und so eine fortdauernde periostale Blutversorgung des Frakturbereiches erlauben.
Um die Frakturheilung zu unterstützen, werden zunehmend lokal Wachstumsfaktoren eingesetzt, die den Heilungsund Umbauprozess aus der zusätzlich einzubringenden Spongiosa beschleunigen. Als weitere supportive Maßnahmen werden die Behandlung mit Bisphosphonaten (Osteoporosemittel), die Stoßwellentherapie, endogende Ultraschalltherapie sowie elektromagnetische Bestrahlung diskutiert [6].

Infolge des bereits vorgeschädigten Knochens kann bezüglich der Frakturheilung nach Folgefrakturen kein Regelzeitintervall angegeben werden. Die ggf. notwendige Metallentfernung sollte ausschließlich nach klinischen und radiologischen Gesichtspunkten erfolgen, also bei blanden Wundverhältnissen erst dann, wenn radiologisch sicher eine ausreichende Frakturheilung stattgefunden hat. Meist hat zur Beurteilung der Durchbauung einer komplizierten Fraktur die Computertomographie eine wesentlich höhere Aussagekraft als die konventionelle Röntgenuntersuchung. Häufig kann und sollte das Osteosynthesematerial belassen werden.

\section{Prophylaxe}

Zur Vermeidung septischer Verläufe und der Entstehung bakterieller Knocheninfekte ist im operativen Bereich stets auf gewissenhaftes Einhalten der Hygienemaßnahmen zu achten.

Single-shot-Antibiotikumgabe bei Osteosynthesen und Endoprothesenimplantationen sowie die Antibiotikumtherapien bei der Versorgung offener Frakturen sind mit hoher Evidenz nachgewiesen wirksam. Bei der Osteosynthese osteitisbedingter Refrakturen ist die intraoperative Prophylaxe mit einem testgerechten Antibiotikum, bezogen auf den ehemaligen Infektionserreger, sinnvoll.

\section{Schlussfolgerungen}

Pathologische Frakturen im Rahmen bakterieller Knochenentzündungen sind in der Regel Rezidivfrakturen im durch die Infektion geschädigten Bereich. Seltener ist die direkte infektbedingte Destruktion des Knochens.

Im Rahmen infektbedingter Knochendestruktionen und dadurch bedingter Frakturen, wie bei der Osteomyelitis, steht zunächst die Sanierung des Infektes im Vordergrund. 
Ursächlich für eine Folgefraktur ist die inkomplette Regeneration des Knochens mit konsekutiver Minderbelastbarkeit. Es handelt sich um ein multifaktorielles Geschehen. Ermüdung, Neutralisation, Versprödung usw. treten regelhaft kombiniert auf. Einer der wesentlichen Faktoren bei der Entstehung von Folgefrakturen ist die Dystrophie, die als führende Ursache für septisch bedingte Refrakturen gilt und in erster Linie durch die infektbedingte Durchblutungsstörung hervorgerufen wird.

Neben Zonen der verminderten Knochenbildung finden sich bei der Infektdystrophie Zonen der Sklerosierung und der knöchernen Hypertrophie. Als Folge fokaler Ernährungsstörungen resultiert ein oft ungerichteter, diffuser und funktionell minderwertiger Knochenaufbau [7,8].

Therapeutisch stehen bei den postinfektiösen Folgefrakturen Maßnahmen zur Stabilisierung und Weichteilsanierung im Vordergrund. Neben Plattenosteosynthesen (winkelstabil), Marknagelungen und Spongiosaplastiken kommt den plastischen Weichteildeckungen eine wesentliche Bedeutung zu.

Entscheidend für die Nachbehandlung ist eine konsequente Dystrophieprophylaxe.

Durch stabile Osteosynthesen können Ruhigstellung und Immobilisation auf ein Minimum reduziert werden. Über krankengymnastische Frühmobilisation, differenzierte, zunehmende Teilbelastungen und durch die von dem Patienten eigenständig durchzuführende Muskelkräftigung kann der inaktivitätsbedingten Dystrophie erfolgreich vorgebeugt werden.

\section{Literatur}

1 Bischoff M, L Kinzl, A Schmelz. Die komplizierte Wunde. Unfallchirurg 1999; 102: 797 804

2 Eggers C, D. Wolter. Autologe Spongiosaplastik in der Behandlung der chronischen Osteomyelitis. Unfallchirurgie Nr. 2 1986; 12: 104-106

3 Fleischmann W, E Lang, M Russ. Infektbehandlung durch Vakuumversiegelung. Unfallchirurg 1997; 100: 301-304.

${ }^{4}$ Höntzsch D. Die Knocheninfektion als Ursache der pathologischen Fraktur. Langenbecks Arch Chir Suppl II Kongressbericht 1989

${ }^{5}$ Hofmann GO, T Bär, V. Bühren. Osteosyntheseimplantat und früher postoperativer Infekt: Sanierung mit oder ohne Materialentfernung? Chirurg 1997; 68: 1175-1180

${ }^{6}$ Lengsten $\mathrm{AL}, \mathrm{SH}$ Ralston. Management of Paget's desease of bone. Rheumatology (Oxford) 2004, Aug; 43 (8): 955-959. Epub 2004 June 8.

7 Meeder PJ, T Hase und K Wagner. Folgefrakturen des Femurschaftes, Chirurg 1993; 64: 918-925

${ }^{8}$ Mitchell DC. Fractures in brittle bone deseases. Orthop Clin North Am. 1979 Nov 3; 3: 787-792

${ }^{9}$ Musero LM, CN Mcharo. Chronic Osteomyelitis: continueing orthopedic challange in developing countries. Int Orthop 2001; 25; 2: $127-131$
${ }^{10}$ Mutschler W, R Wirbel: Pathologische Frakturen. Unfallchirurg 1997; 100: 410-429

11 Offjong Bassey L. Pathologische Frakturen als Komplikation von Osteomyelitis. Unfallchirurg 1987; 90: 304-398

12 Rolauffs B, TM Bernhardt, C von Eiff, ML Hart, D Bettin. Osteopetrosis, femorale fracture and chronic osteomyelitis caused by staphylococcus aureus small colonie variants (SCV) treatet by girdlestone dissection - six-yearfollow-up. Arch Orthop Trauma Search 2002 Dec; 122 (9-10): 547-550

13 Stein T.v. Vakuumtherapie und die hyperbare Sauerstofftherapie. In: Willy C.: Die Vakuumtherapie. C.Willy, Ulm 2005; S. 329

${ }^{14}$ Trinkhaus K, S Wenisch, C Siemers, D Hose, R. Schnettler. Bohrmehl, eine Quelle vitaler Zellen! Erste Ergebnisse von humanen Proben. Unfallchirurg 2005; 108: 650-656

\section{Dr. med. Thomas von Stein}

Chirurg/Unfallchirurg

Assistenzarzt Abtlg. für Sept. Chirurgie Prof. Dr. med. Volker Bühren Ärztlicher Direktor

BG-Unfallklinik Murnau

Prof.-Küntscher-Str. 8

82418 Murnau 